\begin{tabular}{|l|l|l|l|l|}
\hline Philologus & 155 & 2011 & 2 & $307-325$ \\
\hline
\end{tabular}

Gunther Martin

\title{
MEORUM PERICULORUM RATIONES UTILITAS REI PUBLICAE VINCAT ZUR HISTORIZITÄT DER VIERTEN CATILINARIA
}

Die Frage, zu welchem Grade die überlieferten Reden Ciceros den wirklich gehaltenen entsprechen, wird wohl nie exakt zu beantworten sein. Die Bandbreite der Änderungen, die angenommen werden, reicht von einer Neuformulierung unter gelegentlicher Korrektur oder Auslassung von Details bis zu einer gänzlichen Umgestaltung der Argumentation ${ }^{1}$. Nicht zuletzt die unbestreitbare Tatsache, dass Cicero Reden veröffentlicht hat, die nie gehalten wurden, führte dazu, dass man dem geschriebenen Text in der Forschung mit großem Misstrauen begegnete. Cicero selbst berichtet bisweilen von Überarbeitungen seiner Reden (z. B. Att. 1, 13, 5; 13, 20, 2; 15, 1a, 2), und in einigen Reden sind Auslassungen klar gekennzeichnet (z. B. Mur. 57). Dies sind jedoch Probleme, die nicht zu der Annahme berechtigen, Cicero habe eine Rede gänzlich neu geschrieben und seine Strategie und Taktik in der Rede mehr darauf ausgerichtet, was er zum Zeitpunkt der Veröffentlichung habe sagen wollen, als auf das wirklich Gesagte. Dies könnte man nur dann behaupten, wenn eindeutige externe Indizien das Gegenteil bewiesen oder wenn der Text einer Rede unbestreitbar anachronistisch oder unangemessen wäre. Gerade im letzten Punkt ist die Gefahr groß, dass ein subjektiver Maßstab angelegt und Unangemessenheit konstatiert wird, wo sich bei genauerem Hinsehen eine Erklärung anbietet.

Nirgends ist das Problem, welche Teile und Argumente einer Rede man für angemessen oder gar realistisch hält, deutlicher als in den Reden, die sich mit der Catilinarischen Verschwörung befassen. Bei diesen wurde Cicero häufig dafür gescholten, die Situation aus der Sicht der Jahre ab 60 v. Chr. darzustellen, als abzusehen war, dass Clodius nicht ruhen würde, die Exekution der Catilinarier gegen Cicero zu verwenden. Es ist vor allem die letzte Catilinaria, die umfassender Bearbeitung verdächtigt wird, da in ihr das Bewusstsein dessen, was kommen sollte, am ausgeprägtesten scheint. Robin Nisbet schreibt: „The note of artificiality is particularly unwelcome in the Fourth Catilinarian, which dealt with the fate of the prisoners. A decision had to be reached by nightfall, but Cicero finds time for eloquent digressions and unreasonable exempla. He repeatedly alludes to the danger that threatens himself; much of this is prompted by the changed circumstances of $60 \mathrm{~B}$. C. when he had been put on the

1 Vgl. jetzt Manuwald (2007) 54-58 mit ausführlicher Bibliographie. 
defensive“2. Bestimmte Annahmen über Zeitdruck und ein Urteil über die Angemessenheit bestimmter Redeteile führen hier zu der Annahme nachträglicher Umarbeitung ${ }^{3}$. Wie weit diese gehe, darüber gibt Nisbet keine Auskunft.

Dycks neuer Kommentar präsentiert nun die radikalisierte Version eines Vorschlags von Michael Winterbottom: Die Vierte Catilinaria sei eine Komposition aus Äußerungen, die Cicero an verschiedenen Stellen der Debatte getätigt und entsprechend seinen neuen Zwecken umgearbeitet habe. Namentlich $\mathbb{S} 4-6$, 7-10 und 24 stammten aus unterschiedlichen Stadien der Debatte. Nach Winterbottom enthält die Rede einen deliberativen Kern, der jedoch durch den Rahmen einen forensischen Anstrich erhalte ${ }^{4}$.

Im Folgenden soll gezeigt werden, dass die Rede trotz gewisser Zäsuren ein einheitliches Gebilde darstellt, dem ein relativ eng gefasster Platz in der Debatte zugewiesen werden kann. Die Künstlichkeit und die Unangemessenheit, die Nisbet erkennen will, werden erklärbar, wenn die Rede als exhortatio verstanden wird, das heißt als Aufforderung, schnell ${ }^{5}$ eine Entscheidung zu fällen; deliberativ formuliert: die Senatoren sollen sich gegen eine Vertagung und für ein Urteil im Sinne des Staates (nicht zwischen den gestellten Anträgen) entscheiden.

Die Beweiskraft von Ciceros Brief ad Atticum 2, 1, 3 für die Veröffentlichung von Ciceros konsularischen Reden im Jahr 60 wurde bereits 1972 von W. C. McDermott in dieser Zeitschrift widerlegt. Doch unabhängig von der Datierung der Veröffentlichung gibt es gute Gründe, weshalb man die Ansicht aufgeben sollte, die Reden spiegelten die neue Bedrohungslage der Jahre seit 60 wider, und Vorahnungen über Ciceros Zukunft und die Angriffe durch die populare Seite seien erst nach Clodius' Freispruch möglich gewesen. Die Quellen zeigen auf verschiedene Weise, dass Cicero

2 Nisbet (1965) 63.

3 Erinnert sei hier lediglich an die Neubewertung der Wichtigkeit von exempla in den letzten Jahren, die die zentrale argumentative und ethopoietische Rolle historischer Verweise belegt: vgl. z. B. Bücher (2006) 318-22 und zuletzt van der Blom (2010) 12-17.

${ }_{4}$ Dyck (2008) 208, nach Winterbottom (1982) 62. In Winterbottoms Vorstellung geschieht diese Umarbeitung allerdings vor allem wegen des Hungers eines weiteren Publikums nach forensischen Produkten. Dycks Position bleibt vage, was die Form der Komposition angeht: ob die ganze Rede neu geschrieben sei oder aus Versatzstücken bestehe, und wenn Letzteres, ob man in drei Teile ( $\left.\int 1-6,7-23,24\right)$ oder anders teilen müsse. Derartige Thesen sind freilich älter. Schnitzer (1837) 14 hält die ersten sechs Paragraphen ebenfalls für Überbleibsel des Anfangsreferats. Chambalu (1888), sondert nachträglich hinzugefügte Bestandteile aus und rekonstruiert das Anfangsreferat und die Intervention nach Cäsars Rede aus dem Rest; für weitere Rekonstruktionen vgl. Cape (1995) 257. Eine extreme Ansicht wurde zuletzt von Lintott (2008) 17 vertreten: „it may be plausibly argued that the speech from $\$ 7$ onwards is ex post facto invention.“

5 Der Zeitdruck, unter dem der Senat steht, wird nicht etwa, wie Nisbet will, ignoriert, sondern thematisiert: $₫ 6$ celeriter vobis vindicandum est. 
genügend Grund zu der Annahme hatte, eine Hinrichtung der Verschwörer werde auf ihn zurückfallen. Mehr noch: es ist klar, dass Cicero sich dieser Gefahr frühzeitig bewusst sein konnte und auch war.

Die Vierte Catilinaria selbst erwähnt, Cäsar verstehe (intellegit), dass die lex Sempronia, die die Exekution römischer Bürger verbot und so jedem Gegner eine Handhabe gegen den Konsul gab, nicht auf die Verschwörer anwendbar sei ( $\mathbb{1 0}$ ). Die Behauptung, dies könne nur ein argumentatorischer Trick sein und Cicero habe Cäsar dieses Argument untergeschoben ${ }^{6}$, wird dadurch entkräftet, dass die lex Sempronia im öffentlichen Bewusstsein des Jahres 63 durchaus präsent war: Cicero selbst verweist in Pro Rabirio Perduellionis ( $(12)$ auf sie ${ }^{7}$. Die lex Sempronia wurde nach Cassius Dios Bericht im Senat durch Metellus Nepos noch im Dezember 63 als Argument gegen Ciceros Amtsausübung angeführt (37, 42, s.a. 37, 38). Ähnlich wie später Clodius scheint Nepos vor dem Volk die Exekution der Catilinarier zur Stimmungsmache genutzt und den Senat und insbesondere Cicero wegen der Hinrichtung römischer Bürger ohne Volksbeschluss angeklagt zu haben. Die Reaktion Ciceros und des Senats bewegte Nepos' Bruder Celer, zu jener Zeit Proprätor, im Januar 62 dazu, Cicero in einem Brief wenig verhüllt $\mathrm{zu}$ warnen: quae quoniam nec ratione nec maiorum nostrum clementia administrastis, non erit mirandum si vos paenitebit (fam. 5, 1, 2). Die Formulierung clementia ist so weit gefasst, dass man darunter unschwer nicht nur Nepos' Behandlung, sondern auch die Verurteilung der Catilinarier verstehen kann ${ }^{8}$. Selbst innerhalb des Senats mangelte es also offenbar nicht an Kräften, die bereit waren, Ciceros Vorgehen gegen ihn zu verwenden.

Metellus' Brief ist der früheste in den ciceronischen corpora nach der Aufdeckung der Verschwörung. Doch auch die nachfolgenden, abgefasst lange vor dem Bona DeaSkandal, enthalten ähnliche Hinweise: Mitte April 62 beschwert sich Cicero bei Pompeius, dass er ihm nicht zur Rettung des Vaterlandes gratuliert habe. Cicero legt sich und Pompeius die für beide Seiten akzeptable Erklärung zurecht, dieser habe etwas Derartiges in der Öffentlichkeit nicht tun können quod vererere ne cuius animum offenderes ( fam. 5, 7, 3). Er unterlässt jeden weiteren Hinweis, was auch gar nicht nötig ist, da Sender und Empfänger wissen müssen, warum ein Glückwunsch Anstoß erregen könnte. Es könnte sich schlichtweg um ein Zeichen von Parteilichkeit handeln, das die Popularen Pompeius übel nehmen könnten. Doch dafür erscheint die Formulierung zu scharf. Verständlicher wäre eine solche Furcht, wenn die Gratulation als Gutheißung einer Tat wie der Hinrichtung der Verschwörer angesehen würde. Dass dies von Anfang an der größte Stein des Anstoßes für die 'Popularen'

${ }^{6}$ Vgl. Drummond (1995) 44, der aus der Abwesenheit des Arguments bei Sallust schließt, es müsse sich um einen Cäsar von Cicero zugeschriebenen Gedanken handeln. Ob dieses Manöver aus dem Jahr 60 stamme oder schon in der Originalrede vorgenommen worden sei, lässt Drummond offen.

7 Denkbar wäre, dass Cicero auch diesen Verweis nachträglich eingefügt hätte; doch hätte dieser seine Stellung zur Zeit der Veröffentlichung eher geschwächt, da er gewissermaßen seinen Gegnern unnötigerweise Argumente liefert.

8 Vgl. Hoffer (2003) 96. 
war, wird aus dem nächsten erhaltenen Brief klar, abgefasst gegen Ende desselben Jahres (fam. 5, 6). Darin berichtet Cicero me excludunt et aperte vindicem coniurationis oderunt $(\mathbb{S} 2)$.

Der Streit um Cicero begann also sehr früh, und eine Ahnung, dass Gefahr für ihn bestand, darf man einem erfahrenen Politiker wie Cicero vermutlich zubilligen, zumal es einige Hinweise darauf gibt. Zum einen berichtet Cicero selbst in Pro Sulla, dass er ein Protokoll der Senatssitzung vom 3. Dezember anfertigen ließ. Dass dies keine Routine war, sollte aus der Art der Erwähnung klar werden (Sull. 41): vidi ego hoc, indices, nisi recenti memoria senatus auctoritatem buius indici monumentis publicis testatus essem, fore ut aliquando ... aliter indicata baec esse diceret, quo facilius vento aliquo in optimum quemque excitato posset in malis rei publicae portum aliquem suorum malorum invenire. itaque introductis in senatum indicibus constitui senatores qui omnia indicum dicta, interrogata, responsa perscriberent. Selbst wenn das Motiv nachträglich zur Betonung von Ciceros Weitsicht eingefügt worden sein sollte, so dürfte doch kein Zweifel daran bestehen, dass dieses Protokoll tatsächlich existierte und Cicero leicht hätte überführt werden können, wenn er in der publizierten Version der Rede falsche Angaben gemacht hätte.

Zwei Tage später, so erfahren wir von Plutarch, ließ er den Verlauf der Debatte mitstenographieren. Plutarch weist darauf als eine außerordentliche Maßnahme, vielleicht sogar ein echtes Novum hin ${ }^{9}$. Cicero bewies also einigen Einfallsreichtum, um sich abzusichern und keine Gerüchte und falsche Versionen über die Senatssitzung in Umlauf kommen zu lassen.

Zuletzt deutet der bloße Umstand, dass Cicero sich bemühte, die Verschwörer quam maxume manifestos (Sall. Catil. 46, 2) zu ergreifen, und dann eine relatio durchführte, statt sich der Hoheitsgewalt zu bedienen, die er sich mehrfach zugesteht (Catil. 1, 3-4; 9; 27-28; 2, 3; 28; 3, 15) und die er in Pro Rabirio Perduellionis verteidigt, darauf hin, dass er sich seiner Sache nicht sicher war und eine formale Bestätigung seines Vorgehens durch den Senat einholen wollte ${ }^{10}$.

All dies spricht dafür, dass Cicero sehr wohl um die Gefahren wusste, die ihm sein Umgang mit der Verschwörung bereitete. Welches Ausmaß diese Vorahnung genau hatte, lässt sich nicht sagen, doch teilte er sie mit seinen Kollegen: Einige, die popularer Umtriebe besonders verdächtig waren, erschienen erst gar nicht, um sich nicht den Anfeindungen auszusetzen, die sie bei einer Beteiligung an der Abstimmung befürchteten (Cic. Catil. 4, 10) ${ }^{11}$.

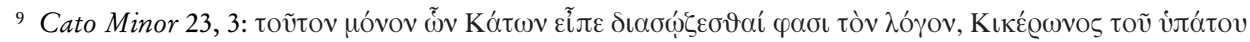

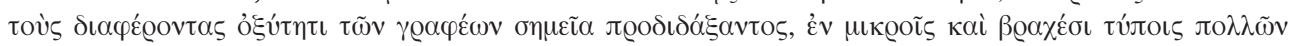

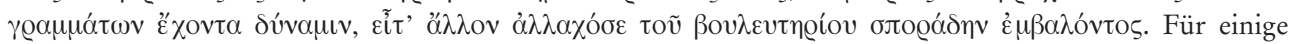
spätere Beispiele vgl. Suet. Iul. 55, 3, Ascon. 42C und generell Boge (1966) 45-47. Berry (1996) ad Sull. 42, 3 nimmt an, es könne sich um dieselben Senatoren gehandelt haben, die schon die vorherige Senatssitzung protokollierten, doch lässt Plutarchs Formulierung eher an Schreiber oder Sekretäre denken.

10 Vgl. Drummond (1995) 11-15; laut Cic. Att. 12, 21, 1 hob Cato diesen Umstand lobend hervor.

$11 \mathrm{Zu}$ deren Identität s. Drummond (1995) 14-15. 
Wenn nun sowohl Cicero selbst als auch der Rest des Senats sich bewusst waren, dass ihr Votum ausgenützt werden konnte, wenn einige der Sitzung deshalb fernblieben (und sich damit auch in eine Position brachten, aus der heraus sie später angreifen konnten), so wird es kaum erstaunen, dass Cicero das Thema aufgreift. Aus welchen Gründen er dies tut, wird noch zu sehen sein. Die Informationen, die wir über die Catilinarien hinaus besitzen, zeigen jedenfalls den Rahmen, innerhalb dessen über Gefahren diskutiert wurde. Und dieser Rahmen lässt eine klare Aussprache über die Risiken einer Hinrichtung als durchaus plausibel erscheinen. Über die vorgebrachten Inhalte schweigen unsere unabhängigen Quellen.

Auch aus den Reden selbst lässt sich ein Anhaltspunkt dafür gewinnen, dass Cicero im Jahre 60 nicht hemmungslos einfügt, was er zu seiner eigenen Verteidigung verwenden kann. Denn dann gäbe es für etliche Stellen in den früheren Reden keine echte Erklärung. Cicero spricht von Anfeindungen, denen er bei einer Bestrafung ausgesetzt sein wird. Doch dies bezieht sich konkret auf die contio, in der der Senatsbeschluss bekanntgegeben wird $(\$ 9,11)$, nicht die künftige Haltung des Volkes. Auch wo Cicero von den künftigen Gefahren für den Staat spricht, erwähnt er nur die Verschwörer, die, sofern man sie nicht vom Gemeinwesen fernhalte, weiterhin versuchen würden, den Staat zu zerstören: eos cum a pernicie rei publicae reppuleris, nec vi coercere nec beneficio placare possis $(\mathbb{2} 2)$. Die Gefahr, die hier beschrieben wird, geht also nicht von einem zukünftigen Rächer von der Art des Clodius aus, sondern von den Vaterlandsverrätern der Stunde. Solche Verweise auf Ereignisse, die entweder inzwischen obsolet sind oder nie eintraten, könnten in einer apologetischen Schrift unterbleiben.

Ähnliches gilt für die ersten beiden Reden. Dort ist der juristische Streitpunkt nicht die Bestrafung der Verschwörer, sondern Ciceros Aufforderung an Catilina, Rom zu verlassen. Sowohl im Senat als auch (in modifizierter Form) vor dem juristisch weniger beschlagenen Volk weist er darauf hin, dass er Catilina nicht ins Exil schicke bzw. geschickt habe (womit er an die Grenzen seiner Amtsgewalt gegangen wäre) ${ }^{12}$. Vor den Senatoren erwähnt er, dass Catilina sich über die scheinbar eigenmächtige Aufforderung zu gehen beschwert: „refer" inquis , ad senatum “; id enim postulas et, si hic ordo sibi placere decreverit te ire in exilium, optemperaturum te esse dicis (1, 20-1).

Vor dem Volk nimmt derselbe Punkt breiten Raum ein (2, 12-16), wobei Cicero betont, dass ihm seine Gegner Vorhaltungen machten: at etiam sunt, qui dicant, Quirites, a me eiectum in exilium esse Catilinam. Catilina selbst indemnatus innocens in exilium eiectus a consule vi et minis esse dicetur ( $\$ 14)$. Cicero zeigt sich hier wiederum in einer juristisch potentiell bedenklichen Lage, mit der Gefahr, dass die popularen Aufwiegler seinen Kopf fordern könnten, wenn er seine Macht als Konsul in unkonstitutioneller Weise missbrauchte.

12 Vgl. $\$ 13$, Mur. 6, Dyck ad Catil. 1, 20, 4-7. 
Der große Unterschied zur Hinrichtungsdebatte besteht darin, dass das Argument nach 63 nie von einer „Opposition“ gegen Cicero verwendet wurde. Cicero beweist dieselbe Wachsamkeit gegenüber möglichen Attacken seiner Gegner wie in der vierten Rede - sie stellt sich aber in diesem Fall als unbegründet heraus. Wäre es Cicero also darum gegangen, sich mit den Catilinarien gegen Anwürfe späterer Jahre zu verteidigen, wäre die Betonung von Catilinas freiwilligem Rückzug unnötig gewesen. Andernfalls sollten wir akzeptieren, dass er die Gefahren sah und benannte.

Die detaillierte Rekonstruktion der Senatsdebatte wird vor allem dadurch erschwert, dass keine Quelle einen umfassenden Bericht enthält. Außer Frage steht, dass der consul designatus Silanus als erster aufgerufen wurde und einen Vorschlag machte, der zumindest so aufgefasst wurde, als fordere er die Todesstrafe. Dem folgten die anwesenden Konsulare (Att. 12, 21, 1). Der designierte Prätor Cäsar setzte einen Antrag dagegen, der die Internierung in den Municipien vorsah. Sallust berichtet von einem Antrag Tiberius Neros, der eine Verschiebung der Debatte ins Spiel brachte (Catil. 50, 4, so auch Appian civ. 2, 1, 5), bevor Cato mit einer flammenden Rede die Mehrheit für die Exekution sicherte. Die offizielle Form von Neros Vorschlag, dessen genauer Zeitpunkt sowie die Reaktionen darauf sind umstritten ${ }^{13}$ : Von Silanus wird einerseits berichtet, er habe sich Neros Vorschlag angeschlossen (Sall. Catil. 50, 4), andererseits soll er seinen eigenen Antrag so uminterpretiert haben, dass

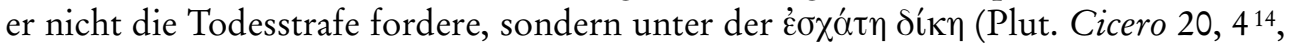
oft gedeutet als Übersetzung von summum supplicium) vielmehr lebenslange Haft verstehe.

Der Teil, der als Kern von Ciceros Rede gilt, lässt sich recht eindeutig lokalisieren. Die Debatte ist noch in vollem Gange, und Cicero schließt nicht aus, dass noch weitere Anträge gestellt werden: video duas adhuc esse sententias. Wir befinden uns also eindeutig nach der Rede Cäsars, da sein Antrag von Cicero paraphrasiert wird (\$\$ 7-8). Gleichzeitig hat Silanus seine Meinung nicht modifiziert, da Cicero die unzweideutige Formulierung morte esse multandos verwendet. Es ist auch deutlich, dass Nero keinen Antrag gestellt hat, andernfalls könnte Cicero seinen Vorschlag bei der Paraphrase der Anträge nicht einfach unterschlagen, dabei aber das Kernelement des Vorschlags als Bemerkung außerhalb der regelkonformen Debatte darstellen $(\mathbb{1} 14)$ :

13 Ryan (1998) 118 Anm. 151-3 diskutiert die einzelnen Möglichkeiten und zeigt, wie sich die einzelnen Quellen widersprechen. Dass Nero vor Cäsar zu Wort kam, lässt sich aufgrund der senatorischen Rangfolge und Att. 12, 21, 2 ausschließen. Auch Ciceros Rede lässt für ein solches Szenario keinen Platz. Plutarch, der seinen Bericht vermutlich hauptsächlich aus Cicero schöpft (vgl. Pelling (1985) 312-322), spricht von Silanus' Modifikation, aber nicht von einer vorhergehenden Stimmabgabe Neros (Plut. Cicero 21, 3; Cato Minor 22, 6).

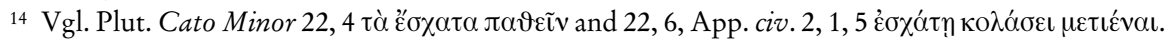


iaciuntur enim voces, quae perveniunt ad auris meas eorum, qui vereri videntur, ut habeam satis praesidii ad ea, quae vos statueritis hodierno die, transigunda. (Was diese Formulierung bedeutet, wird noch zu klären sein.) Und selbst wenn Neros Äußerung nicht als echte sententia zu gelten hätte, da sie nicht direkt die Frage de poena quid censeatis ( $(6)$ beantwortet, könnte Cicero einen Antrag, der von einem designierten Konsul unterstützt wird, kaum so missachten.

Nach der Rede fährt Cicero mit der sententia fort (\$20): nunc, antequam ad sententiam redeo, de me pauca dicam. Dycks Erklärung, dass Cicero so die divisio einleite („,before he calls for a vote“, ad loc.), kann nicht stimmen, während Fuhrmann in seiner Übersetzung („bevor ich auf den Beschlussantrag zurückkomme“) den Sinn korrekt widergibt: Cicero nimmt die Befragung der Senatoren, die er durch seine Rede unterbrochen hat, wieder auf. Der Ausdruck selbst hat keine Parallele, doch man beachte den Unterschied, den Cicero beispielsweise zwischen ad rem redeo und ad rem venio macht. Ersteres bezeichnet die Rückkehr zum Thema nach einer Abschweifung, Letzteres den Übergang zu einem neuen Punkt ${ }^{15}$. Cicero leitet also an unserer Stelle nicht eine neue Handlung ein, sondern fährt mit derjenigen fort, die er mit seiner Stellungnahme unterbrochen hat: Innerhalb der Rede geschieht kein Zeitsprung zur Abstimmung, sondern die Befragung wird beim nächstniedrigeren Senator fortgesetzt. $\$ 7(a d h u c)$ und die Worte in eigener Sache am Ende werden also in derselben Situation gesprochen.

Schwieriger ist die Bestimmung der Lage zu Beginn. $\$ 6$ scheint auf den ersten Blick am besten an den Anfang der Debatte zu passen: sed ego institui referre ad vos, patres conscripti, tamquam integrum, et de facto quid iudicetis, et de poena quid censeatis. illa praedicam, quae sunt consulis. ego magnum in re publica versari furorem et nova quaedam misceri et concitari mala iam pridem videbam, sed hanc tantam, tam exitiosam haberi coniurationem a civibus numquam putavi. nunc quicquid est, quocumque vestrae mentes inclinant atque sententiae, statuendum vobis ante noctem est. Doch wenn Cicero die Kurie bereits mit dem Plan für eine relatio betreten hat, macht es keinen Unterschied für institui, an welchem Punkt er spricht (vgl. instituistis, $\mathbb{2} 24$ ). Bei dem Wort praedicam sollten wir von Dyck, der es auf den dem namentlichen Aufruf der Senatoren vorausgehenden Bericht bezieht, Abstand nehmen. In diesem Fall hätte Cicero im Anschluss an $\mathbb{} 6$ die Senatoren über die Entwicklungen und Einzelheiten informiert, bevor er zum Auftakt der eigentlichen Debatte die Frage de poena gestellt hätte ${ }^{16}$. Cicero hätte nicht nur einen zeitlichen Sprung zwischen seinen Reden kaschiert und einen Teil ausgelassen, sondern vor allem auch einen Leerverweis in seiner Rede stehen lassen und die ausgelassene Partie nicht wie andernorts ${ }^{17}$ markiert. Im Gegensatz dazu hat schon Halm das Richtige gesehen ${ }^{18}$ : Cicero verweist mit praedi-

\footnotetext{
15 De orat. 2, 351; fin. 1, 65; Tusc. 5, 63; fam. 2, 10, 2 und viele andere gegen off. 3, 99; Att. 14, 16, 1; 16, 15, 1.

${ }_{16} \mathrm{Zu}$ diesem Teil der Sitzung vgl. Bonnefond-Coudry (1989) 472-474.

17 Vgl. den ersten Absatz.

18 (1856) ad loc.: "praedicam bezieht sich besonders auf die Forderung, ut ante noctem statuatur.“
} 
cam vielmehr auf die unmittelbar folgenden Sätze: Was er als Konsul zu sagen hat und sagen darf, ist, dass der furor der Verschwörung jede Vorstellungskraft überstiegen hat ${ }^{19}$ und der Senat nun sein Urteil fällen soll. Die Einschätzung de facto und de poena bleibt dagegen ganz den Senatoren überlassen. Dies entspricht einem Leitmotiv der Rede: Mehrfach betont Cicero, dass er seine Kompetenzen nicht überschreitet - wohl nicht zuletzt, weil er sich von einem Teil der Verantwortung zu befreien hofft ${ }^{20}$.

Im größeren Zusammenhang wird die Bedeutung von $\ 6$ klar. In $\ 5$ hatte Cicero hervorgehoben, dass die Senatoren die Schuld der Festgenommenen bereits konstatiert hätten. Die Betonung des nächsten Satzes liegt nun nicht auf institui referre, sondern auf quasi integrum: „Trotzdem habe ich es unternommen, die Sache so vor Euch zu bringen, als gäbe es kein solches Präjudiz." Der Senat hat nämlich ein Anrecht darauf, dass ihm nicht ein fait accompli vorgesetzt wird (vgl. Catil. 3, 7). quae sunt consulis ist als weitere Anerkennung der Kompetenz des Senats und der Zurückhaltung des Konsuls zu verstehen.

\section{III}

Die Schwierigkeiten der Kommentatoren sind einerseits auf Probleme mit der Gliederung zurückzuführen, andererseits darauf, dass nicht mehr in Frage gestellt wurde, dass Cicero die Rede im Lichte des Jahres 60 mit politischer Motivation revidiert hatte, und deshalb eine Kompilierung verschiedener Teile leichter akzeptiert wurde ${ }^{21}$. Im folgenden soll gezeigt werden, dass die Rede in sich geschlossen ist und argumentativ das Ziel verfolgt, die Senatoren zu einer Abstimmung über die Strafe zu bewegen.

Die Rede ist nicht typisch deliberativ in dem Sinne, dass Cicero die Senatoren von einem bestimmten Antrag überzeugen wollte. Eine solche Empfehlung liegt, wenn überhaupt, erst nachrangig in Ciceros Absicht. Cicero hat seine Präferenz weitgehend verklausuliert, um seinen unparteiischen Status als vorsitzender Konsul nicht zu gefährden ${ }^{22}$. Im Gegenteil versucht er dafür zu sorgen, dass überhaupt eine schnelle Bestrafung vorgenommen und einer der beiden Anträge angenommen wird.

19 Dies entspricht einerseits dem „effacement“ der Magistrate in der Senatsdebatte, vgl. BonnefondCoudry (1989) 574-89. Andererseits erlaubt es natürlich Cicero darüber hinaus, Verantwortung auf den Senat zu übertragen.

20 Cape (1995) 263-264. Das Motiv der Senatsverantwortung zieht sich auch durch Ciceros spätere Reden: vgl. Robinson (1994) 45-48.

21 Einen extremen Fall stellt Helm (1979) 211-246 dar, wo überhaupt nicht mehr versucht wird, eine auf das Jahr 63 passende Erklärung für die Bestandteile der Rede zu finden. Stattdessen wird für jedes mögliche Argument der Grund genannt, weshalb Cicero es eingefügt oder weggelassen habe.

22 Cape (1995) 266-269 versucht zu zeigen, wie Cicero seine Präferenz erkennen lässt. Doch zum einen stellen die Bedenken der Senatoren, wie hier nachgewiesen werden soll, für ihn das Hauptproblem dar, zum anderen scheinen Capes Argumente übermäßig spitzfindig (vgl. Anm. 30) und übersehen den relativ deutlichen $\ 22$. 
Die Rede ist eine exhortatio zur Überwindung von Widerständen durch Senatoren, die eine Bestrafung der Catilinarier bzw. eine Abstimmung darüber, aus welchen Gründen auch immer, verhindern wollen. Bis auf die Vorstellung der Anträge in $\mathbb{S} \mathbb{S} 7-8$ können alle Teile der Rede unmittelbar mit den Kategorien Paränese und Widerlegung von Hinderungsgründen (also refutatio der apotreptischen Argumente) erklärt werden. Der folgende Aufbau des Textes soll hier begründet und erläutert werden:

1-6 Anfangsappell: Eine schnelle Entscheidung ist nötig.

7-8 Narratio: Paraphrase der bisherigen Vorschläge

9-23 Argumentatio: Es gibt keine Gründe gegen ein Urteil.

9-13 Refutatio I: Beide Vorschläge sind geeignet.

9-10 Cäsars Vorschlag ist Ausdruck staatsfreundlicher Gesinnung.

11-13 Silanus' Antrag ist nicht unverhältnismäßig grausam.

14-17 Refutatio II: Die Stärke der Unterstützer gewährleistet die Durchsetzung des Beschlusses.

18-19 Comprobatio: Die Verantwortung der Senatoren

20-23 Refutatio III: Cicero benötigt keine Rücksichtnahme

24 Schlussappell: Cicero wankt nicht, und die Senatoren sollten es ebenfalls nicht.

Cicero schweift demnach nicht, wie häufig kritisiert, zur (für die Abstimmung irrelevanten) Heroisierung der eigenen Person ab. Entscheidend ist die Einordnung dessen, was hier als argumentatio bezeichnet wird. Nach Dyck stellen $\mathbb{\$} \$ 9-10$ und $\int \mathbb{S} 20-22$ Exkurse zur Unterscheidung zwischen wahren und falschen Popularen bzw. $\mathrm{zu}$ Ciceros Position dar. Cape ${ }^{23}$ sieht $\$ \$ \$ 6-10$ als Darlegung der Anträge und $\$ \mathbb{\$} 11-13$ als Argumentation, warum Silanus' Antrag scheinbar strenger, gegenüber dem Gemeinwesen aber milder (und daher vorzuziehen) sei.

Doch sollte schon die strenge Parallelität der beiden Abschnitte vor der Abtrennung von $\mathbb{S}$ 9-10 von 11-13 warnen. Cicero weist beidesmal eingangs in ähnlichen Gedanken darauf hin, dass die Annahme von Silanus' Antrag seine Position gegenüber dem Volk (d. h. in der contio des nächsten Tages) schwerer gestalten mag, dass dies aber keine Rolle spielen soll ( $\$ 9$ mea video quid intersit ${ }^{24}$. si eritis secuti sententiam C. Caesaris ... sin illam alteram ...; $\mathbb{1} 11$ sive hoc statueritis ... sive Silani sententiam sequi malueritis ...). Anschließend gibt Cicero die Begründung für die Tauglichkeit des jeweiligen Antrags.

23 (2002) 151-2.

${ }^{24}$ mea interest sollte in diesem Zusammenhang nicht als „es ist in meinem Interesse“ (OLD s.v. intersum $9 \mathrm{~b}$ „it is of advantage, it is expedient") übersetzt werden, sondern als „es macht einen Unterschied für mich“ (idem $8 \mathrm{~b}$ „it makes a difference, it is of importance, it matters“). Cicero erklärt nämlich im Anschluss, dass er bei der Annahme von Silanus' Vorschlag keinen populären Unterstützer hätte, sich dafür aber auf die relative Milde des Gesetzes berufen könne. 
Cäsars Antrag empfiehlt sich durch seine staatstreue Gesinnung: habemus enim a Caesare ... sententiam tamquam obsidem perpetuae in rem publicam voluntatis, die ihn von denen unterscheidet, die nur dem Wort nach populares sind; sein Strafantrag wird als besonders streng dargestellt ${ }^{25}$. Seine Position erscheint also als die eines wahrhaft am Staatswohl orientierten, die Stimmungen nicht ausnutzenden Mannes von dignitas. Sein Antrag ist daher ernstzunehmen und steht im Einklang mit der von Cicero zum Leitprinzip erhobenen salus reipublicae.

Nachdem zu Beginn von $\$ 11$ die Alternativen zum zweiten Mal einander gegenübergestellt wurden, geht Cicero über zur Behandlung von Silanus' Antrag und der Widerlegung, dass dieser grausam sei. Vielmehr müsse jedes Einschreiten gegen die Barbarei der Verschwörer als Akt von humanitas und misericordia gewertet werden ( $(11)$. Grausamkeit wird durch Verweis auf die Verhältnismäßigkeit der Strafe in Anbetracht der außerordentlichen Schwere der Tat zurückgewiesen und Silanus’ Antrag als einer beschrieben, den Cicero vor der contio verteidigen konnte.

\S 9-13 bilden also eine Einheit, von der die Zurückweisung des Arguments, Silanus' Antrag sei grausam, nur ein Teil ist. Im Ganzen widerlegt dieser Abschnitt nämlich mögliche Einwände gegen beide vorliegenden Anträge: Cäsars Antrag könnte den Hardlinern zu populistisch bzw. im schlechten Sinne popular erscheinen, derjenige des Silanus durch seine Grausamkeit zu wenig Rücksicht auf die Stimmung im Volk nehmen. Cicero versucht dagegen zu zeigen, dass beide Anträge für die Senatoren akzeptabel sind und es keinen Grund für Ausflüchte gibt.

Die Forschung tendiert zu der Annahme, Cicero werte Cäsars Vorschlag gegenüber Silanus' ab. Unbestritten ist, dass er eine Exekution bevorzugte, und er drückt dies in einem Brief an Atticus auch aus ${ }^{26}$. Doch erst gegen Schluss der Rede - in anderem Zusammenhang - impliziert er einmal, dass der Staat nur sicher sei, wenn man die Verschwörer aus der Welt schaffe (s. u. zu $\ 22$ ). Bei der Zusammenfassung der Anträge verbietet sein konsularisches Ethos solche Ungleichgewichte. Die zuletzt von Cape vorgebrachten Argumente nehmen den Text weniger ernst als die vermeintlichen Botschaften zwischen den Zeilen: Zum einen ist die Erwähnung von Cäsars Argument, dass eine schnelle Hinrichtung milder sei als lange Gefangenschaft, nicht ironisch ${ }^{27}$. Es wird nämlich auch in den Reden bei Sallust erwähnt, wo sich Cato genötigt sieht, Cäsars Ansicht zurückzuweisen (Catil. 52, 13 gegen 51, 20) ${ }^{28}$. Cicero selbst beruft sich auf Cäsars epikureisch beeinflusste Vorstellung, wenn sie ihm rheto-

25 Zum Inhalt vgl. Drummond (1995) 37.

${ }^{26}$ Dies muss die Bedeutung von quod denique ante quam consulerem ipse indicaverim (Att. 12, 21, 1) sein: Zur Bedeutung von consulere als Eröffnung der relatio vgl. Bonnefond-Coudry (1989) 472, pace Shackleton Bailey (1966) ad loc.

27 In (2002) 152 gibt Cape den Gedanken der Ironie auf und behauptet stattdessen, Cicero stelle die Exekution als die natürliche Strafe dar. Doch Ciceros Beweisziel ist negativ: nicht, dass die Exekution der bessere, sondern dass sie ein gangbarer Weg sei.

28 Drummond (1995) 43 übersieht, dass dasselbe Argument auch Cic. Catil. 4, 11 zugrundeliegt. 
risch gelegen kommt $\left(\mathrm{Clu}\right.$. 171) ${ }^{29}$. Es handelt sich zudem nicht um den einzigen philosophischen Gedanken in der Debatte (s.u.), weshalb man Cicero keine Ironie unterstellen sollte, die letztlich Cäsar ganz unkonsularisch der Lächerlichkeit preisgegeben hätte.

Zum anderen, so Cape, verrate die Formulierung uterque et pro sua dignitate ... in summa severitate versatur eine Abstufung zwischen Silanus als consul designatus und Cäsar als praetor designatus, die Ersterem (und vor allem seinem heimlichen Anhänger, dem Konsul selbst) größeres Gewicht gebe ${ }^{30}$. Doch selbst wenn man die dignitas eines Mannes so leicht bemessen könnte, übersieht Cape, dass es sich bei Cäsar um den frischgewählten pontifex maximus handelt, was ihm zusätzliches Gewicht verleihen muss.

Im Vordergrund steht in den $\$ \mathbb{S} 9-13$ also nicht der Vergleich der beiden Anträge, sondern ihrer beider Validität: Solange sie nur einen der beiden Anträge annehmen, wählen die Senatoren in jedem Fall richtig, da sie dem Staat dienen und durch Tradition und Autorität ${ }^{31}$ bzw. durch die aufrechte Gesinnung des Antragstellers Gewicht erhalten. Die Tendenz der gesamten Stelle ist also negativ, auf Widerlegung von Bedenken ausgelegt.

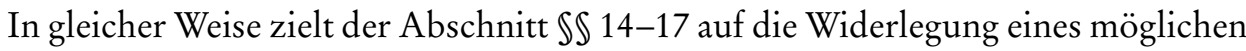
Einwands: dass die Mittel zur Durchsetzung der Staatsgewalt unzureichend seien. Dies entspricht dem von Sallust zitierten Antrag Tiberius Neros. Cicero bringt die Idee eines Bundes der Gutgesinnten (in $\$ 22$ als conspiratio bonorum omnium bezeichnet) ins Spiel, indem er postuliert, dass außer den unwürdigen Elementen des Staates alle Bevölkerungsgruppen zur Unterstützung des Senates bereitstehen ${ }^{32}$.

$\mathbb{S}$ 18-19 bilden anschließend die Wendung ins Paränetische, ein positives Argument nach der Zurückweisung der Einwände der Gegenseite: Aus der Bereitschaft der Bevölkerung, den Staat zu schützen und dem Senat zu helfen, erwächst der Appell an die Senatoren, ihrerseits der Verantwortung gerecht zu werden und der Aufforderung der niederen Schichten nachzukommen ${ }^{33}$.

Auch dieser längere Abschnitt ist also unzweifelhaft argumentatorischer Natur. Er verbindet die Widerlegung von Hinderungsgründen (\$S 14-17) mit der Aufforderung, aktiv zu werden.

${ }^{29}$ Für weitere Diskussion des Topos vgl. die Kommentare vom Ramsey und Vretska ad Catil. 51. 20.

30 Cape (1995) 267-268.

31 Vgl. Bücher (2006) 241-2.

$32 \$ 14$ omnia et provisa et parata et constituta sunt ... omnes adsunt omnium ordinum bomines, omnium generum, omnium denique aetatum, gefolgt von einer Liste aller Stände (außer dem senatorischen) bis hinab zu den Sklaven.

${ }_{33} \mathbb{1} 18$ supplex manus tendit patria communis, vobis se, vobis vitam omnium civium, vobis arcem et Capitolium ... commendat. 
Große Probleme macht nach dieser eindeutigen Passage der nächste Teil (\$\$ 20-23), der wie ein Exkurs de me ipso angekündigt wird ${ }^{34}$. Tatsächlich aber enthält er dieselben Grundaussagen wie die übrige argumentatio, das wesentliche Merkmal ist nur, dass diesmal Cicero selbst der vermeintliche Hinderungsgrund ist. Der Einwand, man gefährde Cicero mit einem Urteil, ist an dieser Stelle nicht ausgesprochen, er wird aber im Eingangssatz der Rede als non-verbal vorgebrachtes Argument impliziert: video vos non solum de vestro ac rei publicae, verum etiam, si id depulsum sit, de meo periculo esse sollicitos $(\mathbb{1} 1)$. Die refutatio muss also belegen, dass die Senatoren ihm nicht nur nicht schaden, wenn sie die Verschwörer verurteilen, sondern im Gegenteil einen Dienst erweisen. Dies tut er, indem er die patriotisch-philosophische Karte des Römers spielt, der durch sein vixi ausdrückt, dass er alles erreicht hat ${ }^{35}$. Er definiert sich als imperator togatus ${ }^{36}$ - auf einer Stufe mit den großen römischen Feldherrn ${ }^{37}$. In einer Debatte, in der Cäsar argumentieren konnte, dass der Tod kein Übel, sondern eine Erlösung sei, ist der Gedanke, dass Ruhm und Nachruhm mehr zählen als eine Verlängerung des Lebens, kaum überraschend ${ }^{38}$.

Vor diesem Hintergrund bedroht eine strenge Haltung des Senats Ciceros Leistung nicht, im Gegenteil: Eine inkonsequente Haltung des Senats, gar eine Verschiebung des Urteils, würde nur den Staat schwächen; die Bedrohung und die politische Auseinandersetzung würden nicht aufhören, wenn man die Verschwörer nicht oder milde bestrafte: eos cum a pernicie rei publicae reppuleris, nec vi coercere nec beneficio placare possis. Schwäche von Seiten des Senats würde nur riskieren, dass Ciceros Verdienst als Retter des Vaterlandes durch dessen späteren Fall zunichte gemacht würde

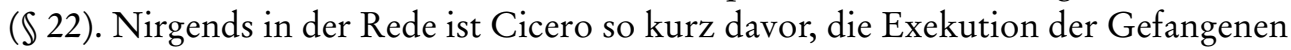
zu fordern, wie hier: Würde man Cäsars Antrag folgen, würden die unversöhnlichen Feinde der Republik am Leben bleiben, wenngleich weggesperrt.

Mit dem letzten Teil wird auch die Funktion der Rahmenpartien deutlich: Es besteht keine Veranlassung, darin lediglich einen Appell an die Römer im Jahre $60 \mathrm{zu}$ sehen, der in der echten Rede deplaziert gewesen wäre. Im letzten Paragraphen wird die exhortatio vielmehr auf den Punkt gebracht: decernite diligenter ... ac fortiter. Cicero hat alle Hinderungsgründe widerlegt und fordert, ganz im Sinne der

${ }^{34}$ Eine „discussion of himself and his virtues as consul“ Cape (2002) 152.

$35 \$ 20$ vitae tantam laudem, quanta vos me vestris decretis honestastis, nemo est adsecutus.

36 May (1988) 58.

37 Die Möglichkeit, dass Eroberungen mehr bedeuten könnten als die Rettung Roms selbst, ist unschwer als ironisches understatement ( $\$ 22$ nisi forte, vgl. Kühner-Stegmann II 416) identifizierbar.

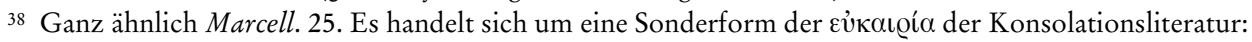
Weniger das durch den frühen Tod ersparte Leid steht im Vordergrund (vielleicht hörte der Römer das mit), sondern die Unmöglichkeit der Steigerung, vgl. Tusc. 1, 109; fin. 3, 45; Sen. dial. 11, 2, 6; epist. 61, 4; s. a. zu möglichen stoischen Ursprüngen Kassel (1958) 82-83, Johann (1968) 114-115. Der Gedanke wird vorweggenommen in $\mathbb{\$}$ 3. Eine deutliche Anspielung darauf findet sich in Phil. 2, 119, in der eben dieser Grundsatz nochmals in aller Kürze herausgehoben wird: etenim, si abhinc annos prope viginti hoc ipso in templo negavi posse mortem immaturam esse consulari, quanto verius non negabo seni. 
Konsulwürde, dazu auf, nur das Staatswesen zu bedenken. Der Verweis auf sich ist nicht Selbstlob, das der Leserschaft des Jahres 60 v. Chr. eine „heroic aristeia“ oder „personal concern“ 39 suggerieren soll, sondern die erneute Versicherung seiner „Dienstbarkeit“ gegenüber dem Senat (babetis eum consulem, qui ... parere vestris decretis non dubitet) und nimmt den Anfang von $\$ 18$ auf, in dem Cicero zum Handeln im Sinne des Vaterlands aufruft. Der Hauptbetroffene dient als argumentum a maiore und Vorbild für kompromissloses und unzögerliches Handeln.

In der gleichen Weise handelt es sich bei den ersten Paragraphen um den paränetischen Auftakt und die Vorstellung dessen, was im Rest der Rede belegt wird. Die Gefahr für Cicero, die die Senatoren daran zu hindern scheint, frei im Interesse des Gemeinwesens zu sprechen und abzustimmen, dominiert. Zu Beginn wie am Ende lautet die Argumentation: Wenn man nicht auf das Beste entscheide, werde die ganze Republik (und damit auch Cicero und seine Familie) den Verschwörern zum Opfer fallen. Der dringende Aufruf zum Handeln, der Appell, nicht hinter die bereits gefassten Beschlüsse zurückzugehen ${ }^{40}$, ist also der Kern der Passage: quare, patres conscripti, incumbite ad salutem rei publicae, circumspicite omnes procellas, quae inpendent, nisi providetis ( $(4)$.

Vor diesem Hintergrund tritt die Bedeutung des $\ 6$ zurück, der der „analytischen“ Interpretation als Hauptindiz dient. Er stellt vielmehr den Übergang dar zwischen der Feststellung, dass der Senat selbst sich über den Sachverhalt und die Dringlichkeit der Sache im klaren ist, und der Zusammenfassung der bisher vorliegenden Beschlussmöglichkeiten. Aufgrund seiner Amtspflicht behandelt Cicero die Sache als res integra-nicht weil sie das wäre, sondern weil er die übliche Form wahren und dem Senat seine Entscheidungsfreiheit belassen muss. Aus der Deklaration und dem Beginn der relatio, wie sie die Forschung interpretiert hat, wird also vielmehr eine eingeschobene Entschuldigung: Cicero hat getan, quae sunt consulis - er hatte keine andere Möglichkeit.

Drei Leitmotive durchziehen also die Rede: die Bedenken um Ciceros Sicherheit, die Erfordernisse des Staates und die Notwendigkeit für die Senatoren, mit Entschlossenheit zu einem Urteil zu kommen. Der erste Aspekt stellt nach Ciceros Angaben den Anlass für die Rede dar und muss zurückgewiesen werden; der zweite ist das Kriterium, das anstelle der Rücksichtnahme auf Cicero berücksichtigt werden muss; der dritte schließlich stellt Ciceros Ziel und die Konsequenz aus der Neuordnung der Prioritäten dar.

Besonders prominent erscheinen diese Punkte in den Rahmenteilen, doch obwohl sie ansonsten gegenüber den konkreten politischen Überlegungen etwas zurücktreten, erstrecken sie sich auch auf die argumentatio. Jene Überlegungen haben jedoch

\footnotetext{
39 Lintott (2008) 148, Winterbottom (1982) 62.

40 S 5: quae sunt omnia eius modi, ut ii, qui in custodiam nominatim dati sunt, sine ulla dubitatione a vobis damnati esse videantur.
} 
aufs Ganze der Rede betrachtet eine untergeordnete Funktion, insofern sie nur die Voraussetzungen und Bedingungen für das entschlossene Handeln des Senats darstellen.

Wenn man die Gesamttendenz der Rede bedenkt, fällt an der Gliederung im Gegensatz zur bisherigen Auffassung weniger die Präsenz von Teilen „in eigener Sache“ auf als vielmehr die große Ausdehnung der negativen Bestandteile: Die Rede wird dominiert von der Widerlegung von Bedenken. Doch dies lässt sich durch Ciceros Stellung als Konsul erklären: Seine Aufgabe beschränkt sich darauf, die relatio ordnungsgemäß durch- und eine Entscheidung herbeizuführen.

Die Absicht der Rede, ebenso wie ihre offizielle Funktion, tritt so zutage: Cicero drängt die Senatoren, zu einem Beschluss zu kommen statt sich zu vertagen oder Ausflüchte für ein mildes Urteil zu suchen. Er wahrt die Unparteilichkeit, die ihm als Konsul ansteht, und es bedarf einiger interpretatorischer Haarspalterei, um eine Abwertung von Cäsars Antrag zu erkennen - ja er lässt die Möglichkeit zu, dass es weitere Anträge geben könnte (adhuc, $\mathbb{S} 7$ ). Aus dem „deliberative kernel“ wird also der argumentative Teil, während der vermeintlich aufgesetzte Rahmen das Ziel und den eigentlichen „Ratschlag“ der deliberativen Rede, d.h. die Paränese zur Entscheidung, enthält.

Warum nun sieht sich Cicero dazu veranlasst, in die Debatte einzugreifen, und zwar in der Art und Weise, wie er es tut? Er reagiert nicht direkt auf Cäsars Antrag, um diesen zu verhindern; es geht ihm eher darum, überhaupt eine Strafe durchzusetzen. Die Gefahr scheint also darin zu bestehen, dass der Senat dazu neigt, den Gedanken an ein endgültiges Urteil aufzugeben. Dies passt nun wiederum am besten zu der Haltung, die als sententia Neros Eingang in Sallusts Werk gefunden hat - eine sententia, die doch, wie oben argumentiert, nicht vorlag.

Ein Hinweis auf die Geschehnisse könnte in der Formulierung liegen, mit der Cicero die Art und Weise beschreibt, wie er von dieser Abstimmungsmöglichkeit erfährt: iaciuntur enim voces, quae perveniunt ad auris meas. Die Bedeutung von ad aures pervenire beschreibt einen eher zufälligen Akt, oft im Zusammenhang mit einem Gerücht (Cic. Verr. II 4, 64; Sull. 12). Derjenige, der hört, lauscht nicht und ist nicht auf Kunde aus, sondern es dringt, ohne dass er es wollte, etwas „an seine Ohren“. Die frühesten Belege in der Dichtung, die etwas später als diese Rede zu finden sind, belegen diesen zufälligen Charakter gut: Lukrez $(6,183-4)$ spricht davon, dass sonus sequitur, qui tardius adlicit auris / quam quae perveniunt oculorum ad lumina nostra. pervenire ist hier verbunden mit den Augen, nicht mit den Ohren, aber es ist unmittelbar davor von einem Ereignis die Rede, das mit einem undefinierten Geräusch, nicht mit verbalem Sinngehalt verbunden ist. Am deutlichsten wird der inzidentelle Charakter der Formulierung in Vergil Aen. 9, 395-6: clamor ad auris / pervenit. 
Cicero hat also von den Bedenken derjenigen, die die Stadt unzureichend gerüstet sehen, nicht in förmlicher Unterredung erfahren, sondern entweder hat ihn ein Gerücht ereilt oder er hat eine Äußerung „aufgeschnappt“, ohne dass er gezielt hingehört hätte. Zu Letzterem passt das abwertende Wort iaciuntur, welches weniger eine wohlgesetzte Aussage zu bezeichnen scheint als vielmehr unartikulierte Laute oder boshafte Drohungen, Klagen oder Beschimpfungen ${ }^{41}$. Ciceros Metaphorik weist auf eine gewisse Unruhe im Senat hin. Der Mangel an Vorbereitung und Wehrhaftigkeit schwirrt nicht als bloße Idee im Raum, sondern wird vermutlich in Zwischenrufen geäußert, die ans Ohr des Konsuls dringen. Cicero greift diese sowie die allgemeine unsichere Stimmung in seiner Rede auf. Sallust könnte einen Hinweis darauf bringen, dass sich auch nach Catos Rede Zwischenrufe dieser Art häuften (Catil. 53, 1): postquam Cato adsedit, consulares omnes itemque senatus magna pars sententiam eius laudant, virtutem animi ad caelum ferunt, alii alios increpantes timidos vocant. Man darf sich darunter vermutlich keine formalen Beiträge vorstellen, eher ein Hin und Her von lose eingeworfenen Kommentaren, bei denen teilweise sogar ein Zwischenrufer dem anderen außer der offiziellen Reihenfolge antwortet: Kritiker von Catos Rede werden ihrerseits geschmäht. Das Abweichen vom Idealfall, bei dem jeder Senator nur dann spricht, wenn er an der Reihe ist, wird für diese Sitzung also von Sallust bestätigt, und jenes Ideal dürfte generell eher eine Wunschvorstellung gewesen $\operatorname{sein}^{42}$. Cicero skizziert also eine Stimmung, ohne sie direkt zu beschreiben. Die Rede präsentiert sich nicht als ein zum Lesen bestimmtes Pamphlet oder eine Meinungsäußerung in einem abgeschirmten Raum, sondern sie gibt die Dynamik des Augenblicks wider. Sie nimmt Bezug auf die Stimmung und auf die Handlungen der Senatoren. Dieselbe unmittelbare Repräsentation der wirklichen Verhältnisse setzt auch der Beginn der Rede voraus ( $\$ 1)$ : Cicero erhebt sich, veranlasst durch die Blicke der Senatoren, die sich - aufgrund der Argumente und Zwischenrufe - auf ihn richten. Der Leser kann sich nur ausmalen, was im einzelnen vor sich ging. Die Atmosphäre ist eine realistische und nicht offensichtlich für den Leser der veröffentlichten Rede entworfen. Letztendlich sind diese beiläufigen Hinweise auf den Verlauf der Sitzung nur für diejenigen wirklich nachzuvollziehen und verständlich, die im Senat anwesend waren und die Reaktionen, Äußerungen und Gesten der Senatoren selbst sahen und hörten.

${ }^{41}$ Lucr. 4, 578; 5, 1081 (Vogelgeschrei), Prop. 4, 9, 32 (der fluchende Hercules). Cicero unterscheidet polemisch zwischen einem geregelten Einspruch vor Gericht und beinahe schon unkontrollierten Einwürfen (Q. Rosc. 25) quin tu hoc crimen aut obice ubi licet agere aut iacere noli ubi non oportet; vgl. Flacc. 6; Sull. 23.

${ }^{42}$ Als selten erreichtes Ideal erscheint das Schweigen außerhalb der Reihe bei Cic. leg. 3, 40. Schweigen der Gegner wird in Cic. ad Q. fr. 2, 3, 3 als bemerkenswert erachtet. Generell zu dem Phänomen vgl. BonnefondCoudry (1989) 505-9, besonders 509: „Les simples clameurs qui ponctuent les séances peuvent, malgré leur caractère peu élaboré, jouer un rôle dans l'elaboration de la décision." Zu den bei Bonnefond-Coudry genannten Beispielen kommt evtl. noch Tac. ann. 14, 45 dissonae voces respondebant. 
Die Gesamtheit dessen, was in der Debatte und in den Zwischenrufen vorgebracht wurde, bleibt verborgen, aber davon, dass auch Rücksichtnahme auf den Konsul gefordert wurde, ist ohne Schwierigkeiten auszugehen. Dass dieser Gedanke dabei jedoch so prominent ist, wie Cicero es beschreibt, ist weder garantiert noch nötig. Helm sieht die Zentrierung auf Cicero als Beleg dafür, dass die Rede überarbeitet wurde: „Sicher richtig ist, daß die unangenehmen Folgen, die Caesar für den Fall der Hinrichtung der Verschwörer androhte, in erster Linie Cicero zu schaffen machen würden. Ganz unwahrscheinlich ist aber, daß die Senatoren, die sich der Meinung Caesars anschlossen [d.h. gegen die Todesstrafe - oder besser, wie wir gesehen haben, überhaupt gegen eine sofortige Bestrafung], so handelten, weil sie den Konsul Cicero schonen wollten und um ihn bangten. Wenn etliche Senatoren für das Gutachten Caesars stimmten, so taten sie dies doch hauptsächlich aus zwei Gründen. Entweder weil sie sich selbst dem Haß der Popularen nicht aussetzen wollten oder weil sie selbst $\mathrm{zu}$ den Popularen gehörten und die ins Auge gefaßten Hinrichtungen für rechtswidrig hielten" 43 .

Die sicher korrekte Feststellung, dass die Bedenken und Äußerungen der Senatoren nicht in erster Linie Cicero galten, bedeutet aber nicht, dass der Konsul den Akzent nicht auf diesen Aspekt hätte legen können oder dürfen. Die Zentrierung auf den Redner selbst, an der sich die meisten modernen Interpreten stoßen, könnte stattdessen auf das Kalkül Ciceros zurückgehen, der sich davon den stärksten persuasiven Effekt versprach.

Cicero präsentiert sich, wie gezeigt, als derjenige, der vorangeht und den Senatoren als Exempel für Furchtlosigkeit den Weg weist. Schon deshalb muss ihm daran gelegen sein, die eigene Todesverachtung möglichst ausführlich darzustellen: nur wenn seine Entschlossenheit die größten Gefahren ignoriert und das Staatswohl höherstellt (meorum periculorum rationes utilitas rei publicae vincat, $\mathbb{\$} 9$ ), wirkt das argumentum a maiore. Die relativ langen Passagen de se ipso sind argumentativ wesentlich und stehen in direktem Zusammenhang mit dem Ziel, die Senatoren zu einer Verurteilung zu drängen.

Eine weitere Funktion der Personalisierung wäre denkbar. Er beschreibt alle Gefahren für den Senat und Anfeindungen gegen die optimatische Politik als gegen ihn selbst gerichtet; er allein ist derjenige, der sich dem Volk im Anschluss stellen muss selbst der Antragsteller begleitet ihn nur zur Unterstützung zur contio. Durch diese Konzentration der Gefahr kann er die egoistischen Bedenken der Senatoren übergehen und sie eines Arguments berauben; er muss also nicht widerlegen, dass es für den Rest des Senats ähnliche Gefahren gibt, sondern kann sie dadurch, dass er sich als

\footnotetext{
${ }^{43} \operatorname{Helm}(1979) 212$.
} 
denjenigen inszeniert, auf den alle Vorwürfe zurückfallen werden, verschweigen ${ }^{44}$ und möglichen Argumenten zur Stellung des Senats gegenüber dem Protest des Volkes oder zu Gefahren für Individuen die Grundlage entziehen. Auch die Rhetorik von der consensio ordinum omnium unterbindet jede Diskussion, die persönliche Voroder Nachteile zum Gegenstand hat ${ }^{45}$.

Der Ansatz, Ciceros scheinbare Abschweifung in eigener Sache für unhistorisch und unmöglich zu erklären, beginnt also am falschen Ende der Interpretation und verkennt bzw. ignoriert die Funktion, die diese Abschnitte im strategischen Aufbau der Rede haben. Die Behauptung, dass für derartiges in einer Senatssitzung wie dieser keine Zeit sei, verkennt gerade den Zweck der Rede: die Debatte effizienter zu gestalten, gewisse Argumente abzuschneiden und eben damit auf eine schnelle Entscheidung zu drängen (ante noctem, celeriter $\$ 6$ ).

Auch die Annahme einer forensischen Umarbeitung erweist sich als überflüssig wie sie ja auch ganz und gar gegen das Ziel der Publikation wäre, das Cicero Atticus gegenüber nennt (Att. 2, 1, 3): fuit enim mibi commodum, quod in eis orationibus quae Philippicae nominantur enituerat civis ille tuus Demosthenes, et quod se ab hoc refrac-

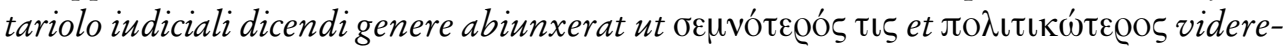
tur, curare ut meae quoque essent orationes quae consulares nominarentur. Es ist der Unterschied zur forensischen Rhetorik und dem Ethos der Gerichtsrede, den Cicero in seinen konsularischen Reden hervorheben will.

Cicero selbst weist darauf hin, dass eine Verhandlung über Verurteilung oder Freispruch nach den vorgehenden Entscheidungen des Senats überflüssig sei - was zu bestimmen sei, sei lediglich die Form der Bestrafung. Auch die Rahmenpartien verteidigen Cicero nicht, sondern porträtieren ihn - und sie porträtieren ihn als einen Mann, der darauf besteht, seine Pflicht zu tun, wobei seine Pflicht gerade aus dem besteht, wofür er später so großem Druck ausgesetzt wurde. Sie sind also ein „Plädoyer“ nicht für den Redner, sondern den Beschluss, und damit Gegenstand einer deliberativen Rede.

Dies schließt selbstverständlich nicht aus, dass die Rede in ihrer publizierten Fassung eine verteidigende Funktion hatte, nur sollte man diese von der Form des veröffentlichten „Produkts“ abstrahieren. Dass Cicero mit der Publikation sein Handeln verteidigen kann, bedeutet nicht, dass sie als solche den Regeln einer Verteidigungsrede folgt. Vielmehr hebt die Veröffentlichung die Rede auf eine Meta-Ebene, auf der ihre Existenz und Form als solche zu Argumenten werden können ${ }^{46}$. Mehrfach hebt Cicero, wie gezeigt, hervor, dass er als Konsul seine Pflicht erfüllt, gleichzeitig seine Rolle aber nicht überschreitet: Er schreibt den Senatoren eben nicht vor, wie sie abzu-

${ }^{44}$ Es ist auffällig, wie die Abwesenheit der Tribunen (s. Anm. 11) beiläufig und ohne Hinweis auf mögliche Gefahren erwähnt wird.

${ }^{45} \mathrm{Zu}$ dieser Argumentation vgl. Konstan (1993) 23-4.

46 Ein ähnlicher Gedanke wird von Jackob (2005) 288-289 im Zusammenhang mit der Miloniana erörtert. 
stimmen haben, sondern nimmt die relatio, also die Befragung des Senats, so vor, als habe jener noch keinerlei Vorfestlegung getroffen. Und er wird jeden Beschluss des Senats mittragen und durchführen (\$6); er nimmt keine Rücksicht auf sich, nur auf die Senatoren als Kollektiv (memorem vestri, oblitum sui) und seine konstitutionelle Rolle (ut mea vox ... officio functa consulari videretur, $\mathbb{S} 19$ ). Die Debatte findet statt, und als ein irrelevantes Argument, nämlich die Gefahren für den Konsul, die Debatte in scheinbar unzulässiger Weise zu beeinflussen droht, greift dieser ein, um derartige Partikularinteressen bei einer Entscheidung über das Staatswohl auszuschließen.

Es ist also die strikt deliberative, dabei aber hinsichtlich der Anträge ostentativ unparteiische Haltung der Rede, in der ihr stärkstes Argument und ihre apologetische Funktion liegen.

\section{Bibliographie}

D. H. Berry, Cicero. Pro P. Sulla Oratio, Cambridge 1996.

H. van der Blom, Cicero's role models. The political strategy of a newcomer, Oxford 2010.

H. Boge, Die Tironischen Noten - die römische Tachygraphie, Altertum 12, 1966, 39-50.

F. Bücher, Verargumentierte Geschichte. Exempla Romana im politischen Diskurs der späten römischen Republik (Hermes ES 96), Stuttgart 2006.

R. W. Cape Jr., The Rhetoric of Politics in Cicero's Fourth Catilinarian, AJPh 116, 1995, 255-277.

R. W. Cape Jr., Cicero's consular speeches, in: J. M. May (Hrsg.), Brill's companion to Cicero. Oratory and rhetoric, Leiden 2002, 113-158.

A. Chambalu, Das Verhältnis der vierten katilinarischen Rede zu den von Cicero in der Senatssitzung vom 5. Dezember 63 wirklich gehaltenen Reden, Programm Neuwied 1888.

M. Bonnefond-Coudry, Le sénat de la république romaine de la guerre d'Hannibal à Auguste. Pratiques délibératives et prise de décision (BEFRA 273), Rom 1989.

A. Drummond, Law, politics and power: Sallust and the execution of the Catilinarian conspirators (Historia ES 93), Stuttgart 1995.

A. Dyck, Cicero. Catilinarians, Cambridge 2008.

K. Halm, Ciceros Reden. Gegen L. Sergius Catilina, Für P. Cornelius Sulla und Für den Dichter Archias, Berlin ${ }^{3} 1856$.

Ch. Helm, Zur Redaktion der Ciceronischen Konsulatsreden, Diss. Bonn 1979.

S. E. Hoffer, Cicero's 'friendly disagreement' with Metellus Celer (Fam. 5. 1-2), SCI 22, 2003, 93-101.

N. Jackob, Öffentliche Kommunikation bei Cicero. Publizistik und Rhetorik in der späten römischen Republik, Baden-Baden 2005.

H.-Th. Johann, Trauer und Trost. Eine quellen- und strukturanalytische Untersuchung der philosophischen Schriften über den Tod, München 1968.

R. Kassel, Untersuchungen zur griechischen und römischen Konsolationsliteratur (Zetemata 18), München 1958.

D. Konstan, Rhetoric and the crisis of legitimacy in Cicero's Catilinarian orations, in: T. Poulakos (Hrsg.), Rethinking the history of rhetoric. Multidisciplinary essays on the rhetorical tradition, Boulder 1993, 11-30.

A. Lintott, Cicero as evidence, Oxford 2008.

G. Manuwald, Cicero. Philippics 3-9 (Texte \& Kommentare 30), Berlin 2007.

W. C. McDermott, Cicero's publication of his consular orations, Philologus 116, 1972, 277-284.

R. Nisbet, The Speeches, in: T. A. Dorey (Hrsg.), Cicero, London 1965, 47-79.

C. B. R. Pelling, Plutarch and Catiline, Hermes 113, 1985, 311-329.

A. Robinson, Avoiding the responsibility: Cicero and the suppression of Catiline's conspiracy, SyllClass 5, 1994, 43-51. 
F.X. Ryan, Rank and participation in the republican senate, Stuttgart 1998.

C. F. Schnitzer, Quaestionum Ciceronianarum particula secunda, Festvortrag Heilbronn 1837.

D. R. Shackleton Bailey, Cicero's letters to Atticus, vol. 5, Cambridge 1966.

K. Vretska, Sallust. De coniuratione Catilinae, Heidelberg 1976.

M. Winterbottom, Schoolroom and Courtroom, in: B. Vickers (Hrsg.), Rhetoric Revalued. Papers from the International Society for the History of Rhetoric, Binghamton 1982, 59-70.

\title{
Universität Bern \\ Institut für Klassische Philologie
}

$\mathrm{CH}-3012$ Bern

\begin{abstract}
This paper proposes a reading of Cicero's Fourth Catilinarian as a consistent and historically realistic attempt at urging the senate to punish the conspirators. Cicero struggles against the senators' inclination to postpone a decision out of fear of the consequences which a severe sentence might have. The speech does not show signs of substantial revision or even contamination of several addresses Cicero made at different stages of the meeting on 5 December 63; nor may the extended passages in which Cicero talks about himself be read as subsequent additions in the light of Clodius' assaults. They rather fit into the exhortative strategy of the speech, forming part of the refutation of any grounds which would allow for a delay of the decision. The extended space they take up is to be explained as a strategy that prevents further discussion of dangers by an argumentum a maiore.
\end{abstract}

Keywords: Cicero, Catilinarian Orations, publication, rhetorical strategy 